\title{
Water-soluble L-cysteine-coated FePt nanoparticles as dual MRI/CT imaging contrast agent for glioma
}

This article was published in the following Dove Press journal:

International Journal of Nanomedicine

23 March 2015

Number of times this article has been viewed

\author{
Shuyan Liang' \\ Qing Zhou' \\ Min Wang ${ }^{2,3}$ \\ Yanhong Zhu' \\ Qingzhi Wu ${ }^{2,3}$ \\ Xiangliang Yang' \\ 'College of Life Science and \\ Technology, Huazhong University \\ of Science and Technology, Wuhan, \\ People's Republic of China; ${ }^{2}$ State \\ Key Laboratory of Advanced \\ Technology for Materials Synthesis \\ and Processing, Wuhan University of \\ Technology, Wuhan, People's Republic \\ of China; ${ }^{3}$ Biomedical Material and \\ Engineering Center of Hubei Province, \\ Wuhan University of Technology, \\ Wuhan, People's Republic of China
}

Correspondence: Yanhong Zhu College of Life Science and Technology, Huazhong University of Science and Technology, Wuhan 430074, People's Republic of China

Tel +86 2787792147

Fax +86 2787792234

Email yhzhu@hust.edu.cn

Qingzhi Wu

State Key Laboratory of Advanced

Technology for Materials Synthesis and Processing, Biomedical Material and Engineering Center of Hubei Province, Wuhan University of Technology, Wuhan 430070, People's Republic of China

Tel +86 278765 I852

Fax +86278788 0734

Email wuqzh@whut.edu.cn

\begin{abstract}
Nanoparticles (NPs) are advantageous for the delivery of diagnosis agents to brain tumors. In this study, we attempted to develop an L-cysteine coated FePt (FePt-Cys) NP as $\mathrm{MRI} / \mathrm{CT}$ imaging contrast agent for the diagnosis of malignant gliomas. FePt-Cys NPs were synthesized through a co-reduction route, which was characterized by transmission electron microscopy, high-resolution transmission electron microscopy, powder X-ray diffraction, Fourier transform infrared spectroscopy, and dynamic light scattering. The MRI and CT imaging ability of FePt-Cys NPs was evaluated using different gliomas cells (C6, SGH44, U251) as the model. Furthermore, the biocompatibility of the as-synthesized FePt-Cys NPs was evaluated using three different cell lines (ECV304, L929, and HEK293) as the model. The results showed that FePt-Cys NPs displayed excellent biocompatibility and good MRI/CT imaging ability, thereby indicating promising potential as a dual MRI/CT contrast agent for the diagnosis of brain malignant gliomas.
\end{abstract}

Keywords: CT, glioma, MRI

\section{Introduction}

Malignant brain gliomas are the fourth most common cause of cancer death because of their extremely high rate of morbidity and mortality. ${ }^{1}$ In the past decade, numerous novel strategies based on various nanomaterials have been developed to conquer the highly mortal brain tumors..$^{2-5}$ Interesting topics in this field include the targeted and controlled delivery system of anticancer drugs, ${ }^{6-9}$ photodynamic, photothermal, and magnetothermal therapy of tumors; ${ }^{10-16}$ and MRI, CT, and fluorescent imaging of brain tumors. ${ }^{17-22}$ Among various nanomaterials, magnetic nanoparticles (NPs) such as superparamagnetic iron oxide NPs (SPIONs) are the most extensively studied. Applications of these SPIONs range from the drug delivery system to MRI contrast agent and magnetothermal therapy for brain gliomas. ${ }^{23-26}$ Thus, studying novel magnetic nanomaterials other than SPIONs for application in the diagnosis and therapy of brain gliomas is of great interest.

FePt NPs with chemically disordered face-centered cubic structure have been well studied because of their promising potential in biomedical applications, including as an anticancer agent and for hyperthermic ablation, magnetic separation of bacteria and biomolecules (such as DNA and proteins), and MRI/CT imaging, due to their excellent chemical stability and magnetic properties. ${ }^{27-29} \mathrm{In}$ particular, the theoretical magnetic moment of FePt NPs is as high as $\sim 1,000 \mathrm{emu} / \mathrm{cc}$, which is much higher than that of iron oxide (300-400 emu/cc). Recently, FePt NPs coated with iron oxides, $\mathrm{SiO}_{2}$, and cysteamine have been demonstrated to have promising potential for application as 
MRI and CT contrast agents. ${ }^{30-33}$ Although good biocompatibility of $\mathrm{SiO}_{2}$ and iron oxides has been suggested, one of the crucial concerns is the possibility of introducing the exogenous coatings into various tissues and organs (especially the brain) when these nanomaterials are used as therapeutic and diagnostic reagents. ${ }^{34-38}$

In our previous work, ${ }^{39} \mathrm{FePt}$ NPs showed high activity in suppressing the proliferation of glioma cells depending on their surface coatings. Lipophilic FePt NPs coated with oleic acid/oleylamine significantly suppressed the proliferation of different gliomas cells (U251, U87, and H4) in time- and dose-dependent manners, but no obvious suppressing effect was observed when glioma cells were treated with L-cysteine (Cys)-coated FePt NPs under the same conditions. Furthermore, FePt NPs coated with either oleic acid/oleylamine or Cys were effectively uptaken by different glioma cells. Therefore, it is interesting to explore the potential of FePt-Cys NPs as a MRI/CT imaging agent for the diagnosis of malignant gliomas. In the present work, the use of Cys-coated FePt NPs as MRI/CT contrast agent was evaluated in three glioma cells (C6, SGH44, and U251). The biocompatibility of FePt-Cys NPs was assessed via 3-(4,5-dimethylthiazol-2-yl)-2,5diphenyltetrazolium bromide (MTT) assay on different cells (ECV304, L929, and HEK293). The results demonstrated that FePt-Cys NPs showed favorable biocompatibility and strong signals for dual MR/CT contrast imaging. Therefore, FePtCys NPs can potentially be utilized as a dual MRI/CT contrast agent for the diagnosis of malignant brain gliomas.

\section{Materials and methods}

\section{Materials}

$\mathrm{K}_{2} \mathrm{PtCl}_{6}, \mathrm{FeCl}_{2} \cdot \mathrm{H}_{2} \mathrm{O}$, and $\mathrm{NaBH}_{4}$ were of analytical grade (Sinopharm Chemical Reagent Co., Ltd, Shanghai, People's Republic of China) and were used without further purification. L-cysteine (Cys) was of biochemical grade. Deionized water $(16 \mathrm{M} \Omega \cdot \mathrm{cm})$ was supplied by a Nanopure water system (Thomas Scientific, Swedesboro, NJ, USA).

The glioma cell lines (C6, SGH44, and U251) and normal cell lines (ECV304, L929, and HEK293) were purchased from the China Center for Type Culture Collection (CCTCC, Wuhan, People's Republic of China) and preserved in our laboratory.

\section{Synthesis of Cys-coated FePt NPs}

FePt-Cys NPs were synthesized according to our previous work. ${ }^{39}$ In brief, $0.0183 \mathrm{~g}$ of Cys $(0.15 \mathrm{mmol}), 0.3651 \mathrm{~g}$ of $\mathrm{K}_{2} \mathrm{PtCl}_{6}(0.75 \mathrm{mmol})$, and $0.0497 \mathrm{~g}$ of FeCl$\cdot \mathrm{H}_{2} \mathrm{O}(0.25 \mathrm{mmol})$ was successively dissolved in $90 \mathrm{~mL}$ deionized water in $\mathrm{N}_{2}$ atmosphere under magnetic stirring. Subsequently, $0.1892 \mathrm{~g}$ of $\mathrm{NaBH}_{4}(5 \mathrm{mmol})$ was dissolved in another $10 \mathrm{~mL}$ of deionized water and added dropwise into the solution containing Cys and metal salts. The reaction was performed for 2 hours. The black precipitate was collected by adding $200 \mathrm{~mL}$ of ethanol and centrifuging at 9,000 rpm for 3 minutes. The product was dried in a vacuum at $30^{\circ} \mathrm{C}$ overnight.

\section{Characterization of Cys-coated FePt NPs}

The phase structure of the sample was identified by powder X-ray diffraction (XRD) on an X'Pert PRO diffractometer (PANalytical B.V., Almelo, the Netherlands) using $\mathrm{Cu} \mathrm{K} \alpha$ radiation $(\lambda=1.5406 \AA)$. The surface information was analyzed using Fourier transform infrared spectroscopy (FT-IR) on a Nexus Fourier transform infrared spectrophotometer (Thermo Nicolet, Waltham, MA, USA). Atomic absorption spectrometry (GBC AVANTA M; GBC Scientific Equipment Pty Ltd., Melbourne, SA, Australia) was used to analyze the molar ratio of $\mathrm{Fe} / \mathrm{Pt}$ in the sample. High-resolution transmission electron microscopy (HRTEM) (JEM-2100F; JEOL, Tokyo, Japan) was used to observe the morphology, the particle size, and detailed information on the lattice of the samples. The size of FePt NPs was measured using a dynamic light scattering instrument (Zetasizer Nano ZS90; Malvern Instruments, Malvern, UK). In order to assess the stability of FePt NPs in solution, the change of particle size was measured in different media (deionized $\mathrm{H}_{2} \mathrm{O}$ and cell culture media) in the period of 7 days.

\section{Relaxivity measurements}

In a typical measurement, the sample was dissolved in deionized water and diluted at an iron concentration range of $0-20 \mu \mathrm{g} / \mathrm{mL}$. The samples were transferred to a 96-well plate, and $T_{2}$ relaxation time was determined by using a whole-body magnetic resonance (MR) scanner (SignaHDx 3.0T; GE Healthcare Bio-Sciences Corp., Piscataway, NJ, USA).

\section{CT imaging capability}

The as-synthesized FePt-Cys NPs were diluted in distilled water at a Pt concentration range of $0-40 \mathrm{mg} / \mathrm{mL}$. Samples were transferred to a 96-well plate. Iohexol was used as the control. CT imaging ability of the FePt-Cys NPs was determined by using the IVIS Lumina XR system.

\section{MRI and CT imaging of Cys-coated FePt NPs in cells}

Different glioma cells (C6, SGH44, and U251) were used to evaluate the MRI and CT imaging ability of FePt-Cys NPs. 
These glioma cell lines were cultured in Dulbecco's Modified Eagle's Medium (DMEM) (Thermo Fisher Scientific, Waltham, MA, USA) supplemented with $10 \%$ fetal bovine serum (Thermo Fisher Scientific) and antibiotics penicillin $(100 \mathrm{U} / \mathrm{mL})$ and streptomycin $(100 \mu \mathrm{g} / \mathrm{mL})$ in a humidified atmosphere with $5 \% \mathrm{CO}_{2}$ at $37^{\circ} \mathrm{C}$.

In a typical MRI measure, glioma cells were incubated with FePt-Cys NPs dissolved in DMEM at different iron concentrations $(0,5,10,15$, and $20 \mu \mathrm{g} / \mathrm{mL})$ at $37^{\circ} \mathrm{C}$ for 3 hours. After that, the cells were washed with phosphatebuffered saline (0.1 M, pH =7.4) three times. Subsequently, the cells were dispersed and suspended in $300 \mu \mathrm{L}$ of agarose gel $(0.5 \%)$. The samples were then quickly transferred to a 96-well plate. MRI imaging was performed by using the 3.0-T whole-body MR scanner.

In a typical CT imaging measurement, glioma cells were incubated with FePt-Cys NPs dissolved in DMEM at different platinum concentrations $(0,2,5,10$, and $20 \mathrm{mg} / \mathrm{mL})$ at $37^{\circ} \mathrm{C}$ for 3 hours. After that, the cells were washed with phosphate-buffered saline $(\mathrm{pH}=7.4)$ three times. Subsequently, the cells were dispersed and resuspended in deionized water. The samples were then transferred to a black 96-well plate. CT images were acquired by using an IVIS Lumina XR system.

\section{Biocompatibility studies}

The cytotoxicity of FePt-Cys NPs was evaluated using different cell lines (ECV304, L929, and HEK293) via MTT assays. In brief, these cells were treated with FePt-Cys NPs at different concentrations $(25,50,75$, and $100 \mu \mathrm{g} / \mathrm{mL} \mathrm{Fe})$ and incubated for different times (24, 48, and 72 hours). The control well contained culture medium without the FePt-Cys NPs. Then, $20 \mu \mathrm{L}$ of MTT ( $5 \mathrm{mg} / \mathrm{mL}$ ) was added to each well and incubated for another 4 hours. Formazan crystal was dissolved in $150 \mu \mathrm{L}$ of dimethyl sulfoxide. The absorbance of each well was measured by a microplate reader (1420 multilabel counter; Perkin Elmer Inc., Waltham, MA, USA) at $490 \mathrm{~nm}$ to determine the relative cell viability.

\section{Results and discussion}

Transmission electron microscopy (TEM) and HRTEM images of as-synthesized FePt-Cys NPs are shown in Figure 1. The homogeneous NPs were obtained with slight aggregation (Figure 1A), which could be attributed to low surface protection. The size of as-synthesized FePt-Cys NPs obtained from TEM image was $\sim 4.8 \pm 0.6 \mathrm{~nm}$. The perfectly aligned lattice planes, as shown in the upper-right inset of Figure 1B, exhibited a well-crystallized structure. The interplanar spacing of $\sim 0.22 \mathrm{~nm}$ obtained from HRTEM image can be ascribed to the adjacent (111) planes of FePt.

XRD pattern of as-synthesized FePt-Cys NPs is shown in Figure 2. The diffraction peaks in XRD pattern were indexed to the (111), (200), (220), and (311) planes of FePt. The diffraction peaks derived from planes (111), (220), and (311) were significantly broadened, which could be attributed to the extremely small size of FePt-Cys NPs. The Fe/Pt molar ratio in FePt-Cys NPs was $\mathrm{Fe}_{28} \mathrm{Pt}_{72}$ according to the atomic absorption spectrometry measurement.
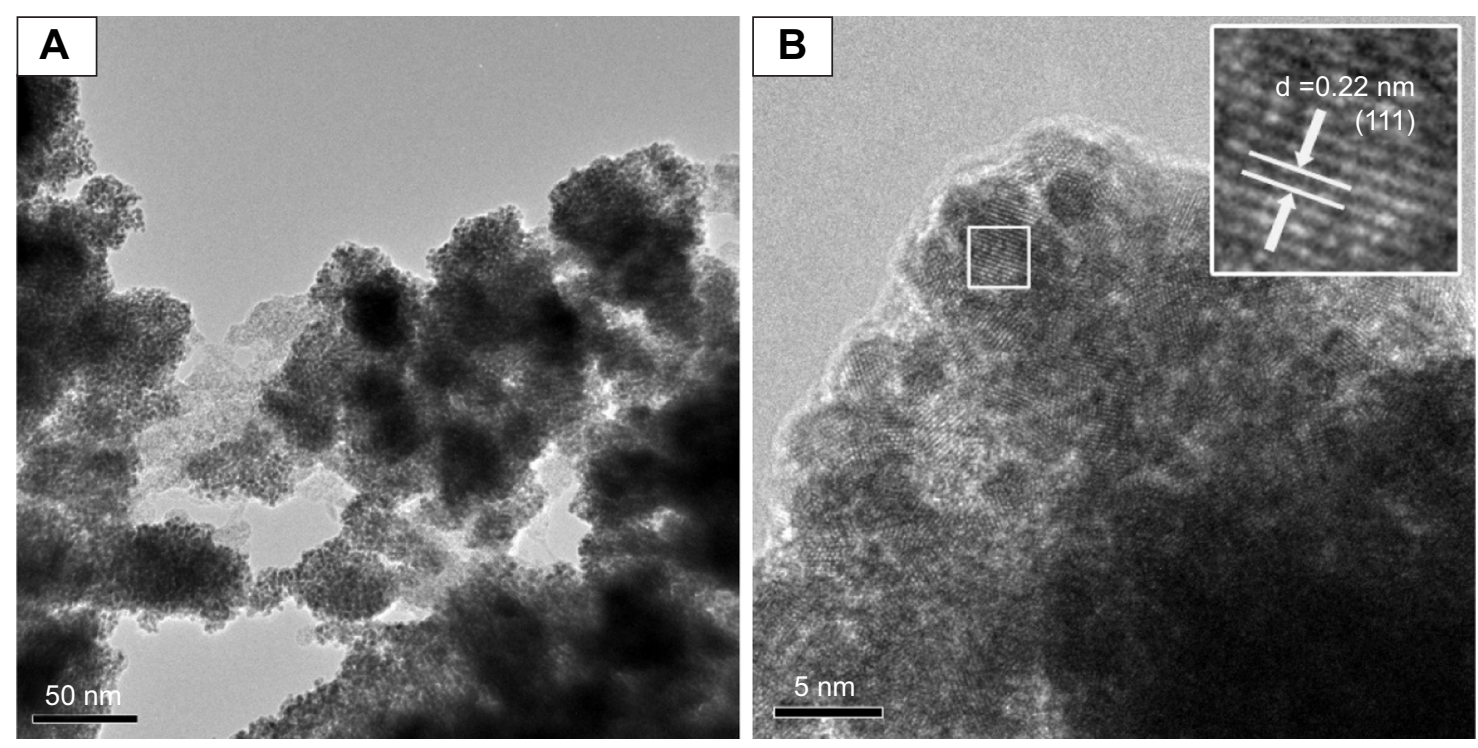

Figure I TEM (A) and HRTEM (B) images of as-synthesized FePt-Cys NPs.

Note: The arrows in the inset of $\mathbf{B}$ indicate the distance between the two adjacent planes.

Abbreviations: $d$, interplanar distance; FePt-Cys, L-cysteine coated FePt; HRTEM, high-resolution TEM; NPs, nanoparticles; TEM, transmission electron microscopy. 


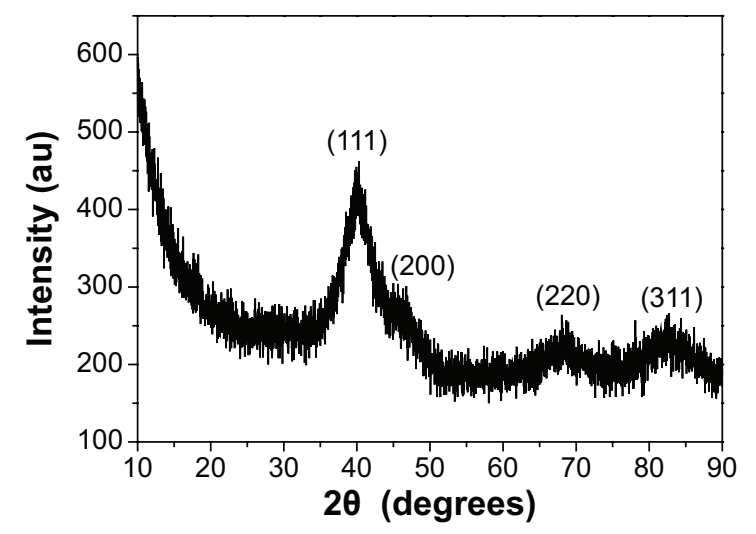

Figure 2 XRD pattern of FePt-Cys NPs.

Abbreviations: FePt-Cys, L-cysteine coated FePt; NPs, nanoparticles; XRD, powder X-ray diffraction.

The surface information of FePt-Cys NPs was characterized by FT-IR spectra. Figure 3 shows the FT-IR spectra of pure Cys and FePt-Cys NPs. The absorption bands at $3,200-2,900 \mathrm{~cm}^{-1}$ and $\sim 1,580 \mathrm{~cm}^{-1}$ in Figure 3A were designated as the stretching vibrations of $\mathrm{NH}_{3}^{+}$and $\mathrm{COO}^{-}$, respectively. These absorption bands were shifted to the higher wavenumbers of $\sim 3,420 \mathrm{~cm}^{-1}$ and $1,605 \mathrm{~cm}^{-1}$ in FT-IR spectrum of FePt-Cys NPs (Figure 3B), respectively, because of the change in the dipole moment when Cys was adsorbed on the surface of NPs with high electron density. ${ }^{40-42}$ It is noteworthy that the absorption at $\sim 2,550 \mathrm{~cm}^{-1}$ derived from the $\mathrm{S}-\mathrm{H}$ stretching vibration of the Cys molecules disappeared in the FT-IR spectrum of FePt-Cys NPs (Figure 3B), indicating the interactions between Cys molecules and FePt NPs because of the high affinity of the mercapto group to the Pt atoms.

A

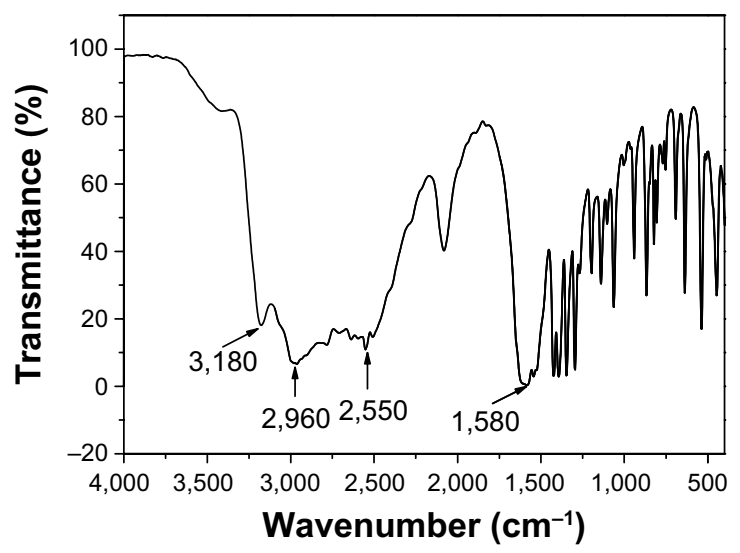

The size distribution and stability of FePt-Cys NPs dispersed in different media were measured. As shown in Figure 4A, the dynamic light scattering hydrodynamic size of FePt-Cys NPs in deionized $\mathrm{H}_{2} \mathrm{O}$ was $\sim 254 \mathrm{~nm}$, which was larger than that obtained from TEM image (Figure 1). The size of FePt-Cys NPs was slightly increased in a cell culture medium (DMEM $+10 \%$ fetal bovine serum) compared with that in $\mathrm{H}_{2} \mathrm{O}$. FePt-Cys NPs could be aggregated together in different media and the accuracy of hydrodynamic size measured by the light scattering method strongly depends on knowing the optical parameters of the particles (such as refractive index and light absorption characteristics). ${ }^{43}$ The stability of FePt-Cys NPs in different media was studied by monitoring the change of hydrodynamic size in the period of 7 days. Figure 4B shows that the hydrodynamic size of FePt-Cys NPs was slightly increased from $\sim 252.3 \mathrm{~nm}$ to $259.1 \mathrm{~nm}$ in $\mathrm{H}_{2} \mathrm{O}$ and from $\sim 258.0 \mathrm{~nm}$ to $267.4 \mathrm{~nm}$ in cell culture media at the end of 7 days, respectively, implying a good stability of FePt-Cys NPs in different media.

The magnetic property of the as-synthesized FePt-Cys NPs was measured by vibrating sample magnetometer at room temperature. Figure 5 shows the characteristic superparamagnetic curve of FePt-Cys NPs. The saturated mass magnetization value was $\sim 16.1 \mathrm{emu} / \mathrm{g}$ Fe, which was approximate to that of cysteamine-coated FePt NPs $(12.3 \mathrm{emu} / \mathrm{g} \mathrm{Fe})$, but was lower than those of $\mathrm{Fe}_{2} \mathrm{O}_{3}^{-}, \mathrm{SiO}_{2}^{-}$, and tetraethylene glycol-coated FePt NPs. ${ }^{30-33}$ The magnetic property of FePt NPs is not only strongly correlated with the degree of chemical ordering of the alloy, but also influenced by the composition and surface coating of the alloy. ${ }^{44} 46$ The surface iron sites are the primary contributors to the magnetization in FePt.

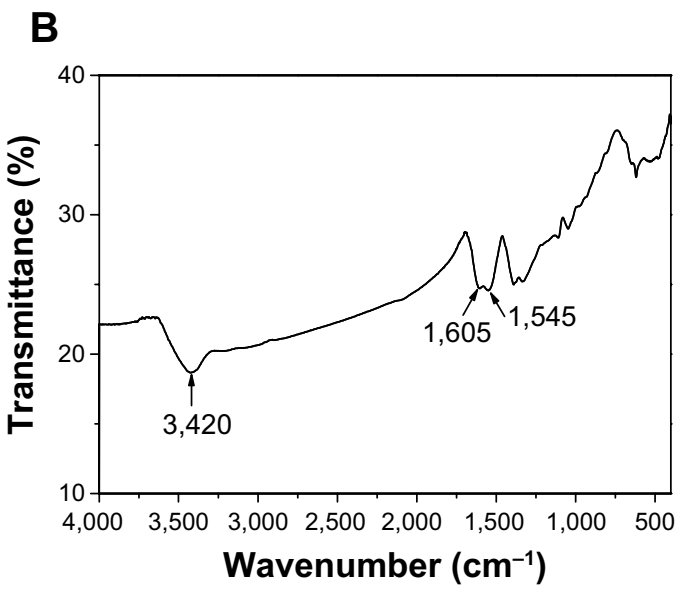

Figure 3 FT-IR spectra of pure Cys molecules (A) and FePt-Cys NPs (B).

Abbreviations: Cys, L-cysteine; FePt-Cys, L-cysteine coated FePt; FT-IR, Fourier transform infrared spectroscopy; NPs, nanoparticles. 
A

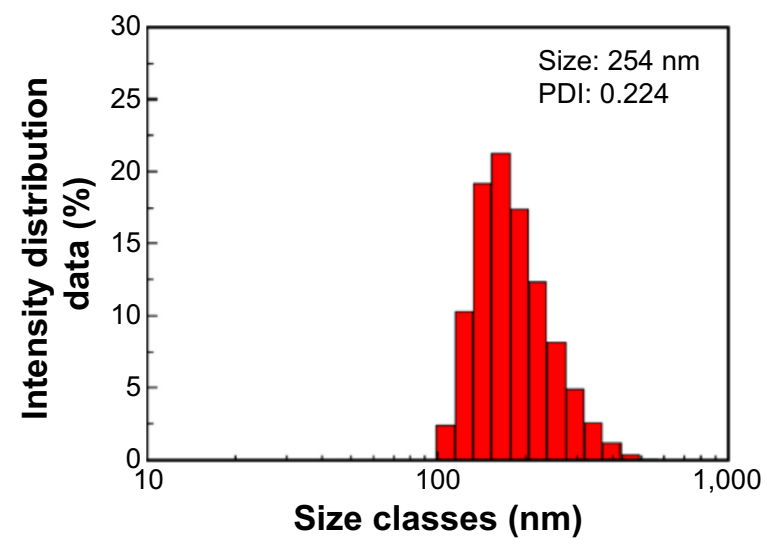

B

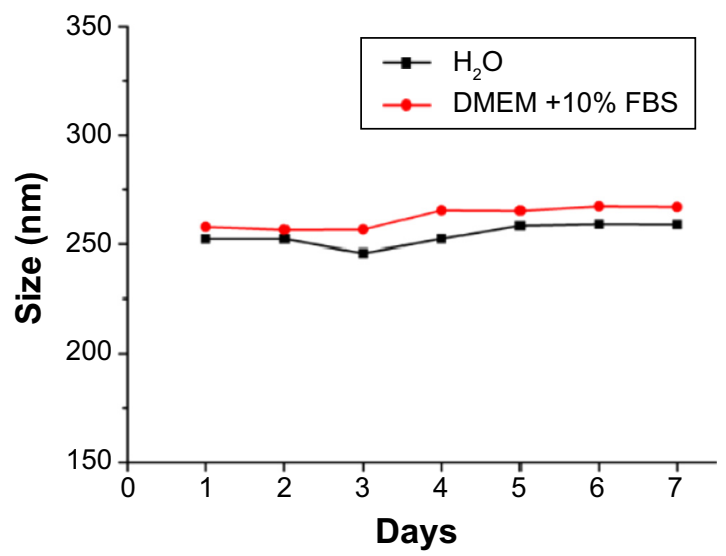

Figure 4 Size distributions of FePt-Cys dispersed in different media for different times.

Notes: (A) Size distribution of FePt-Cys NPs dispersed in $\mathrm{H}_{2} \mathrm{O}$. (B) Size changes of FePt-Cys NPs in $\mathrm{H}_{2} \mathrm{O}$ and cell culture media (DMEM $+\mathrm{I} 0 \%$ FBS) in the period of 7 days. Abbreviations: DMEM, Dulbecco's Modified Eagle's Medium; FBS, fetal bovine serum; FePt-Cys, L-cysteine coated FePt; NPs, nanoparticles; PDI, polydispersity index.

The strong bonding interaction between the surfactant and the surface iron sites results in the decrease of magnetization. For example, theoretical calculations showed that the charge is transferred to the surface iron sites from oleylamine and lowers the atomic magnetization by about $60 \%$ at the iron site in oleylamine-coated FePt NPs. ${ }^{44}$

The $T_{2}$ weighted image for FePt-Cys NPs was examined in a 3.0 T MR imager at various Fe concentrations. Figure 6A shows the $T_{2}$ weighted images of FePt-Cys NPs in the Fe concentration range of $0-20 \mu \mathrm{g} / \mathrm{mL}$ in deionized water. A significant concentration-dependent inverse MR image contrast was observed. Figure 6B shows the linear correlation of the $T_{2}$ relaxation rates $\left(1 / T_{2}\right)$ against the iron concentration. The calculated relaxation rate $\left(r_{2}\right)$ value was $\sim 16.9 \mathrm{mM}^{-1} \cdot \mathrm{s}^{-1}$.

The CT imaging capability of FePt-Cys NPs was evaluated by using an IVIS Lumina XR system. FePt-Cys NPs

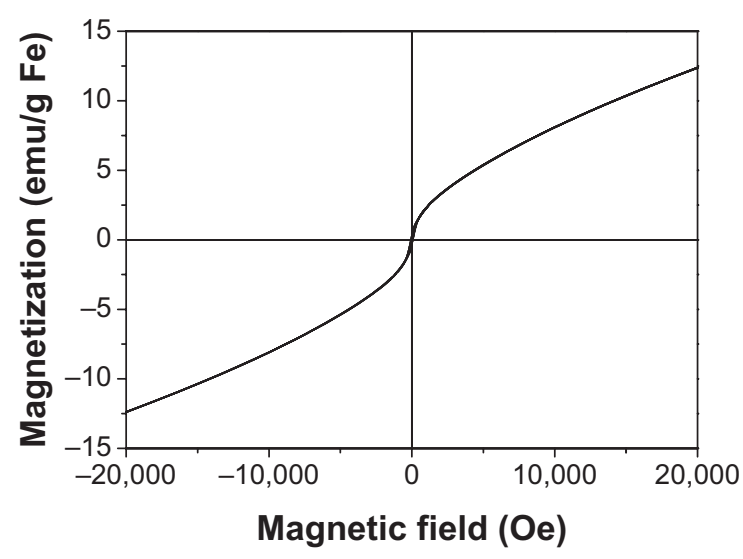

Figure 5 Magnetization hysteresis loop of the as-synthesized FePt-Cys NPs. Abbreviations: FePt-Cys, L-cysteine coated FePt; NPs, nanoparticles. were dissolved in deionized water at a $\mathrm{Pt}$ concentration range of $0-40 \mathrm{mg} / \mathrm{mL}$, and were subsequently transferred to a black 96-well plate. Iohexol at an iodine concentration range of $0-80 \mathrm{mg} / \mathrm{mL}$ was used as the control. As shown in Figure 7A and B, the concentration-dependent enhancement of contrast effect was observed in both iohexol and FePt-Cys NPs. The calculated CT signal contrast intensities were plotted against the concentration of iohexol (Figure 7C) and $\mathrm{Pt}$ (Figure 7D). The CT signal intensity of FePt-Cys NPs at a Pt concentration of $40 \mathrm{mg} / \mathrm{mL}$ was significantly similar to that of iohexol at an iodine concentration of $80 \mathrm{mg} / \mathrm{mL}$. These results

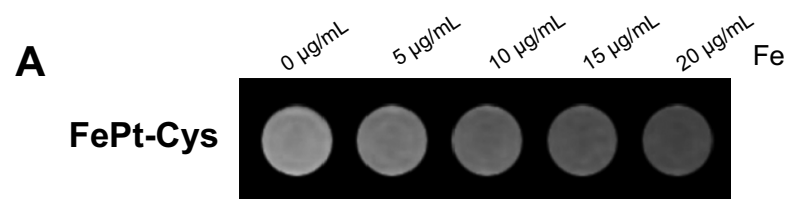

B

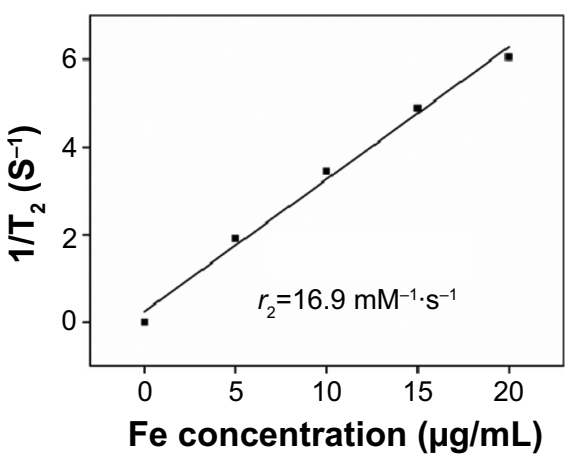

Figure 6 MR contrast imaging of FePt-Cys NPs. Notes: (A) The $T_{2}$-weighted MR images of FePt-Cys NPs at different Fe concentrations. (B) $T_{2}$ relaxation rates $\left(1 / T_{2}\right)$ plotted against the Fe concentration of FePt-Cys NPs. Abbreviations: FePt-Cys, L-cysteine coated FePt; MR, magnetic resonance; NPs, nanoparticles; $r_{2}$, relaxation rate. 
A
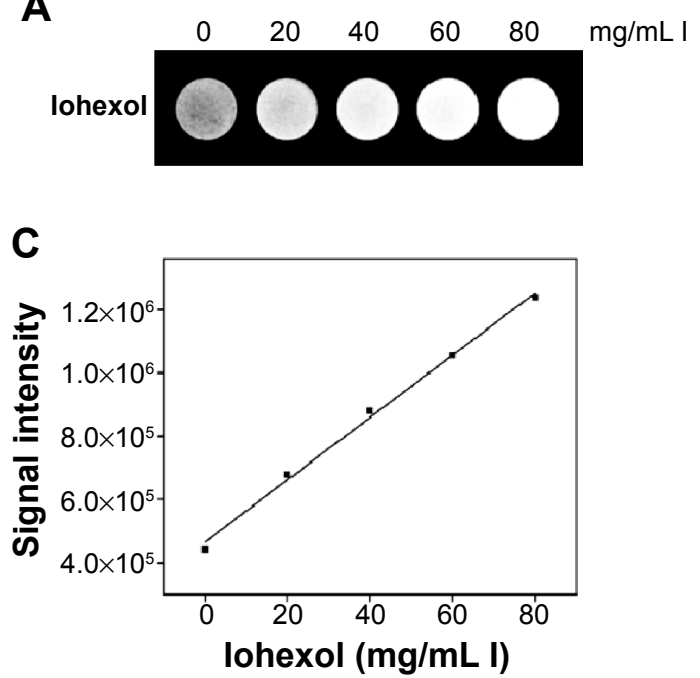

B
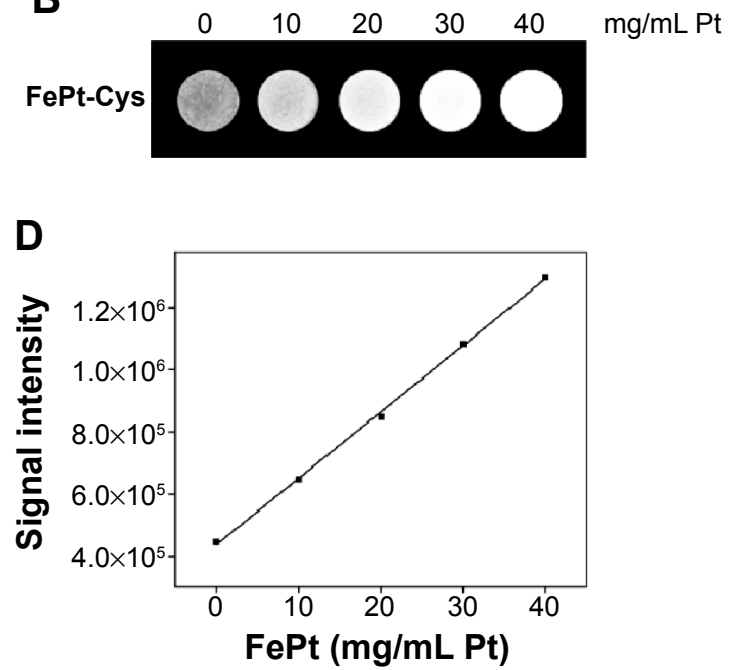

Figure 7 CT imaging evaluation of different samples.

Notes: (A) The CT images of iohexol at different iodine concentrations; (B) The CT images of FePt-Cys NPs at different Pt concentrations; (C) The signal intensity of iohexol plotted against the concentration of iodine; (D) The signal intensity of FePt-Cys NPs plotted against the concentration of Pt.

Abbreviations: CT, computed tomography; FePt-Cys, L-cysteine coated FePt; NPs, nanoparticles.

demonstrated the significantly enhanced CT contrast imaging of FePt-Cys NPs, which was due to the high X-ray absorption coefficient of Pt contained in FePt-Cys NPs. ${ }^{32}$

The MR imaging of FePt-Cys NPs was further evaluated using different glioma cells (C6, SGH44, and U251), as shown in Figure 8A. When FePt-Cys NPs were incubated with $\mathrm{C} 6$ glioma cells at different Fe concentrations, a gradual MR signal decay was observed with increasing of the Fe concentration from $0 \mu \mathrm{g} / \mathrm{mL}$ to $20 \mu \mathrm{g} / \mathrm{mL}$. The Fe concentration-dependent MR signal decay was also observed on SGH44 cells and U251 cells. The CT imaging of FePt-Cys NPs was evaluated using three glioma cells (C6, SGH44, and
U251) at different Pt concentrations. As shown in Figure 8B, a significant enhancement of the gray-scale signal can be observed with increasing Pt concentration on three glioma cells. These results suggested that FePt-Cys NPs could be used as MR and CT contrast agents in glioma cells.

In order to evaluate the biocompatibility of FePt-Cys NPs as an MRI/CT imaging agent, cytotoxicity was measured by MTT assay using different cells (ECV304, L929, and HEK293 cells) as the model. As shown in Figure 9, these cells were treated with FePt-Cys NPs at different concentrations ranging from $25 \mu \mathrm{g} / \mathrm{mL}$ to $100 \mu \mathrm{g} / \mathrm{mL}$ for 72 hours. No significant differences in the viability of these cells were
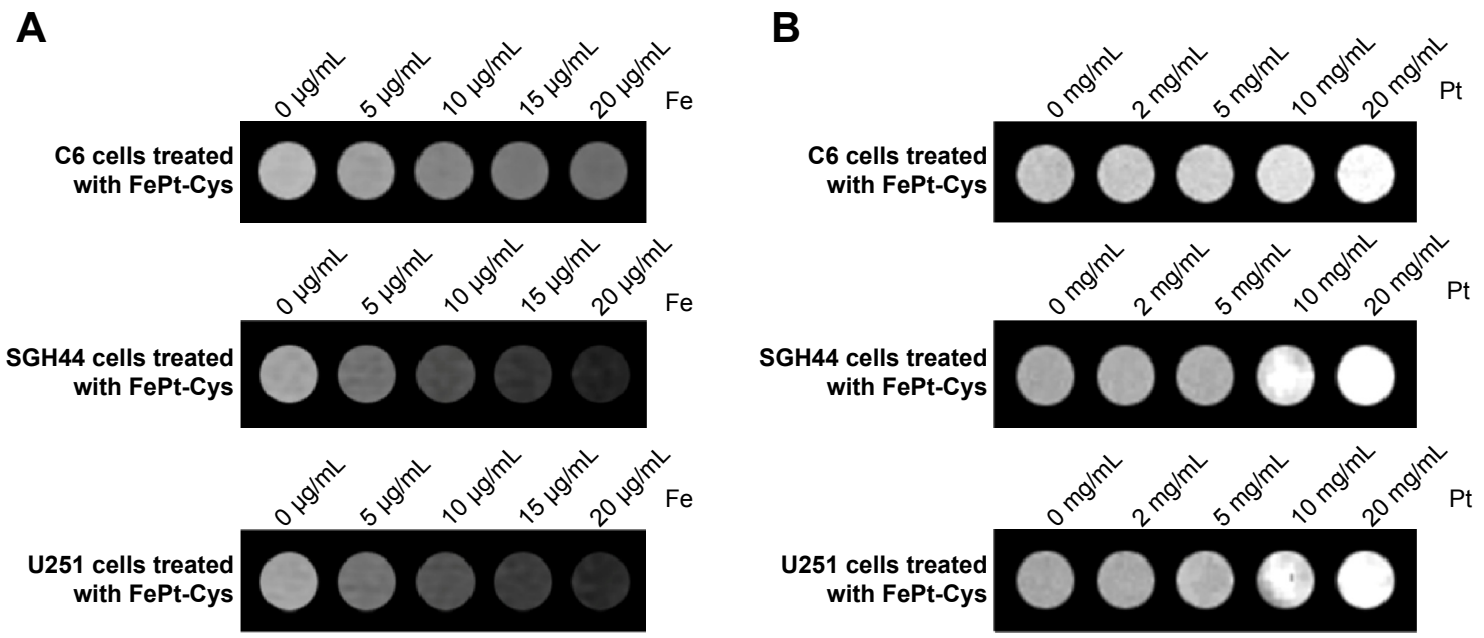

Figure 8 Imaging of different gliomas cells (C6, SGH44, and U25I) treated with FePt-Cys NPs.

Notes: (A) MR imaging of different gliomas cells at different Fe concentrations; (B) CT imaging of different gliomas cells at different Pt concentrations. Abbreviations: CT, computed tomography; FePt-Cys, L-cysteine coated FePt; MR, magnetic resonance; NPs, nanoparticles. 


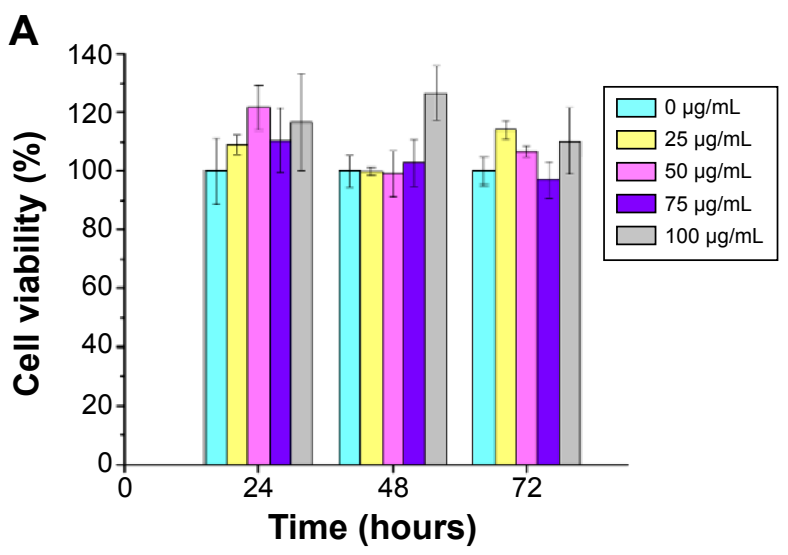

B
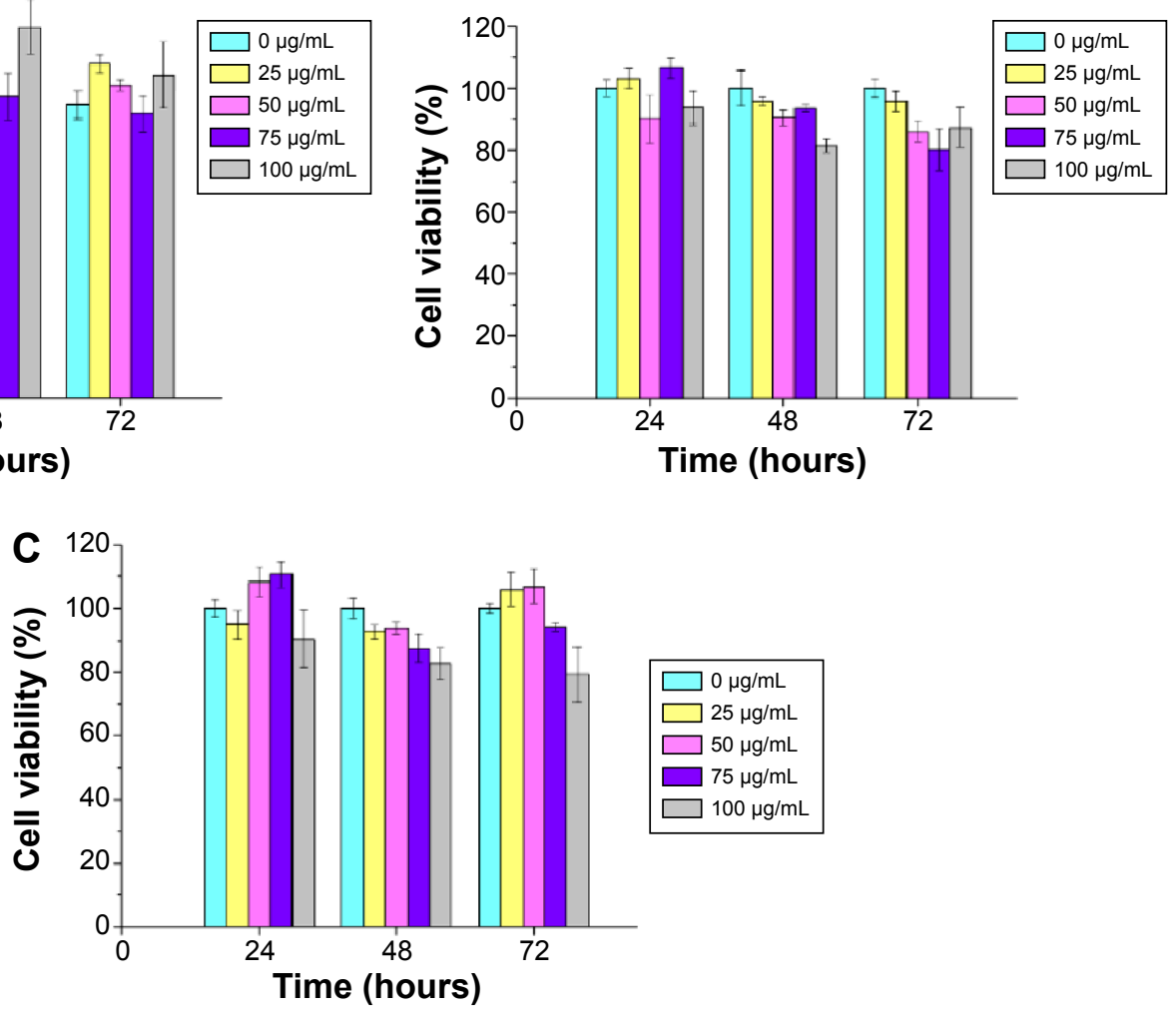

Figure 9 Viabilities of different cell lines treated with FePt-Cys NPs at different Fe concentrations and time intervals.

Notes: (A) ECV 304 cells; (B) L929 cells; and (C) HEK293 cells.

Abbreviations: FePt-Cys, L-cysteine coated FePt; NPs, nanoparticles.

found between the FePt-Cys NP-treated group and the control group. For example, when ECV304 cells were treated with FePt-Cys NPs at a concentration of $100 \mu \mathrm{g} / \mathrm{mL}$ for 72 hours, the viability was $\sim 110.2 \%$ compared with that of the control. Similarly, no significant decrease in cell viability was observed in L929 cells and HEK293 cells treated with FePt-Cys NPs at the same concentrations. These results indicated a good biocompatibility of FePtCys NPs, which is consistent with the results reported previously. ${ }^{39}$

\section{Conclusion}

In summary, the water-soluble FePt-Cys NPs have promising potential as a dual MRI/CT contrast agent for malignant brain gliomas. The CT contrast imaging of FePt-Cys NPs was significantly more sensitive than that of the commonly used iohexol, even if the $r_{2}$ value of FePt-Cys NPs was lower than that of FePt NPs coated by $\mathrm{Fe}_{2} \mathrm{O}_{3}$ and $\mathrm{SiO}_{2}$. Moreover, surface coating of FePt NPs with Cys not only provided good biocompatibility for the FePt-Cys NPs, but also prevented the introduction of unnecessary coatings (such as $\mathrm{Fe}_{2} \mathrm{O}_{3}$, and $\mathrm{SiO}_{2}$ ) that may damage brain tissue. ${ }^{34-38}$ Therefore, based on our preliminary results, FePt-Cys NPs could possibly be used as a dual MRI/CT contrast agent for the diagnosis of brain malignant gliomas. Further optimization of the composition of FePt-Cys NPs is required. In addition, more studies, especially in vivo studies using the animal as the model are needed to further evaluate this agent before it can be developed as a dual-mode imaging agent for clinical application.

\section{Acknowledgments}

We appreciate Ketao Mu and Wenzhen Zhu (Radiology Department of Tongji Hospital, Huazhong University of Science and Technology, Wuhan, People's Republic of China) for great help in the MRI measurement. This work was financially supported by the Natural Science Foundation of China (No 30800256), the basic research project of Wuhan Science and Technology Bureau (No 2014060101010041, No 2014060202010120), the National Basic Research Program of China (973 Program, 2012CB932500), the Keygrant Project of Chinese Ministry of Education (No 313041), and the Fundamental Research Funds for the Central Universities (WUT: 2014-VII-028). 


\section{Disclosure}

The authors report no conflicts of interest in this work.

\section{References}

1. Wen PY, Kesari S. Malignant gliomas in adults. $N$ Engl $J$ Med. 2008;359(5):492-509.

2. Orringer DA, Koo YE, Chen T, Kopelman R, Sagher O, Philbert MA. Small solutions for big problems: the application of nanoparticles to brain tumor diagnosis and therapy. Clin Pharmacol Ther. 2009;85(5): 531-534.

3. Rozhkova EA. Nanoscale materials for tackling brain cancer: recent progress and outlook. Adv Mater. 2011;23(24):H136-H150.

4. Li M, Deng H, Peng H, Wang Q. Functional nanoparticles in targeting gliomas diagnosis and therapies. J Nanosci Nanotechnol. 2014;14(1):415-432.

5. Cheng Y, Morshed RA, Auffinger B, Tobias AL, Lesniak MS. Multifunctional nanoparticles for brain tumor imaging and therapy. $A d v$ Drug Deliv Rev. 2014;66:42-57.

6. Su Z, Xing L, Chen Y, et al. Lactoferrin-modified poly(ethylene glycol)grafted BSA nanoparticles as a dual-targeting carrier for treating brain gliomas. Mol Pharm. 2014;11(6):1823-1834.

7. Chung EJ, Cheng Y, Morshed R, et al. Fibrin-binding, peptide amphiphile micelles for targeting glioblastoma. Biomaterials. 2014; 35(4):1249-1256.

8. Yang ZZ, Li JQ, Wang ZZ, Dong DW, Qi XR. Tumor-targeting dual peptides-modified cationic liposomes for delivery of siRNA and docetaxel to gliomas. Biomaterials. 2014;35(19):5226-5239.

9. Kang T, Gao X, Hu Q, et al. iNGR-modified PEG-PLGA nanoparticles that recognize tumor vasculature and penetrate gliomas. Biomaterials. 2014;35(14):4319-4332.

10. You J, Shao R, Wei X, Gupta S, Li C. Near-infrared light triggers release of paclitaxel from biodegradable microspheres: photothermal effect and enhanced antitumor activity. Small. 2010;6(9):1022-1031.

11. Silva AC, Oliveira TR, Mamani JB, et al. Application of hyperthermia induced by superparamagnetic iron oxide nanoparticles in glioma treatment. Int J Nanomedicine. 2011;6:591-603.

12. Hainfeld JF, Smilowitz HM, O'Connor MJ, Dilmanian FA, Slatkin DN. Gold nanoparticle imaging and radiotherapy of brain tumors in mice. Nanomedicine (Lond). 2013;8(10):1601-1609.

13. Cheng Y, Morshed R, Cheng SH, et al. Nanoparticle-programmed selfdestructive neural stem cells for glioblastoma targeting and therapy. Small. 2013;9(24):4123-4129.

14. Bechet D, Mordon SR, Guillemin F, Barberi-Heyob MA. Photodynamic therapy of malignant brain tumors: a complementary approach to conventional therapies. Cancer Treat Rev. 2014;40(2): 229-241.

15. Yi GQ, Gu B, Chen LK. The safety and efficacy of magnetic nanoiron hyperthermia therapy on rat brain gliomas. Tumor Biol. 2014; 35(3):2445-2449.

16. Mohamed MS, Veeranarayanan S, Poulose AC, et al. Type 1 ribotoxincurcin conjugated biogenic gold nanoparticles for a multimodal therapeutic approach towards brain cancer. Biochim Biophys Acta. 2014; 1840(6):1657-1669.

17. Veiseh O, Sun C, Gunn J, et al. Optical and MRI multifunctional nanoprobe for targeting gliomas. Nano Lett. 2005;5(6):1003-1008.

18. Chertok B, Moffat BA, David AE, et al. Iron oxide nanoparticles as a drug delivery vehicle for MRI monitored magnetic targeting of brain tumors. Biomaterials. 2008;29(4):487-496.

19. Ray A, Wang X, Lee YK, et al. Targeted blue nanoparticles as photoacoustic contrast agent for brain tumor delineation. Nano Res. 2011; 4(11):1163-1173.

20. Xiao N, Gu W, Wang H, Deng YL, Shi X, Ye L. T1-T2 dual-modal MRI of brain gliomas using PEGylated Gd-doped iron oxide nanoparticles. J Colloid Interface Sci. 2014;417:159-165.
21. Ni D, Zhang J, Bu W, et al. Dual-targeting upconversion nanoprobes across the blood-brain barrier for magnetic resonance/fluorescence imaging of intracranial glioblastoma. ACS Nano. 2014;8(2): 1231-1242.

22. Deng $\mathrm{Y}$, Wang $\mathrm{H}, \mathrm{Gu} \mathrm{W}$, et al. $\mathrm{Ho}^{3+}$ doped $\mathrm{NaGdF}_{4}$ nanoparticles as MRI/optical probes for brain gliomas imaging. Journal of Materials Chemistry B. 2014;2:1521-1529.

23. Shevtsov MA, Nikolaev BP, Yakovleva LY, et al. Superparamagnetic iron oxide nanoparticles conjugated with epidermal growth factor (SPION-EGF) for targeting brain tumors. Int $J$ Nanomedicine. 2014;9:273-287.

24. Chertok B, David AE, Yang VC. Brain tumor targeting of magnetic nanoparticles for potential drug delivery: effect of administration route and magnetic field topography. J Control Release. 2011;155(3): 393-399.

25. Xie H, Zhu Y, Jiang W, et al. Lactoferrin-conjugated superparamagnetic iron oxide nanoparticles as a specific MRI contrast agent for detection of brain glioma in vivo. Biomaterials. 2011;32(2):495-502.

26. Sun C, Fang C, Stephen Z, et al. Tumor-targeted drug delivery and MRI contrast enhancement by chlorotoxin-conjugated iron oxide nanoparticles. Nanomedicine (Lond). 2008;3(4):495-505.

27. Gao J, Gu H, Xu B. Multifunctional magnetic nanoparticles: design, synthesis, and biomedical applications. Acc Chem Res. 2009;42(8): 1097-1107.

28. Frey NA, Peng S, Cheng K, Sun SH. Magnetic nanoparticles: synthesis, functionalization, and applications in bioimaging and magnetic energy storage. Chem Soc Rev. 2009;38(9):2532-2542.

29. Ho D, Sun X, Sun S. Monodisperse magnetic nanoparticles for theranostic applications. Acc Chem Res. 2011;44(10):875-882.

30. Gao J, Liang G, Cheung JS, et al. Multifunctional yolk-shell nanoparticles: a potential MRI contrast and anticancer agent. $J$ Am Chem Soc. 2008;130(35):11828-11833.

31. Chen S, Wang L, Duce SL, et al. Engineered biocompatible nanoparticles for in vivo imaging application. J Am Chem Soc. 2010;132(42): 15022-15029.

32. Chou SW, Shau YH, Wu PC, Yang YS, Shieh DB, Chen CC. In vitro and in vivo studies of FePt nanoparticles for dual modal CT/MRI molecular imaging. J Am Chem Soc. 2010;132(38):13270-13278.

33. Yang H, Zhang J, Tian Q, et al. One-pot synthesis of amphiphilic superparamagnetic FePt nanoparticles and magnetic resonance imaging in vitro. J Magn Magn Mater. 2010;322(8):973-977.

34. Koch F, Möller AM, Frenz M, Peiles U, Kuehni-Boghenbor K, Mevissen M. An in vitro toxicity evaluation of gold-, PLLA- and PCLcoated silica nanoparticles in neuronal cells for nanoparticle-assisted laser-tissue soldering. Toxicol In Vitro. 2014;28(5):990-998.

35. Hou Y, Lai M, Chen X, et al. Effects of mesoporous $\mathrm{SiO}_{2}, \mathrm{Fe}_{3} \mathrm{O}_{4}$, and $\mathrm{TiO}_{2}$ nanoparticles on the biological functions of endothelial cells in vitro. J Biomed Mater Res A. 2014;102(6):1726-1736.

36. Bellusci M, La Barbera A, Padella F, et al. Biodistribution and acute toxicity of a nanofluid containing manganese iron oxide nanoparticles produced by a mechanochemical process. Int J Nanomedicine. 2014;9:1919-1929.

37. Sharma HS, Muresanu DF, Patnaik R, Sharma A. Exacerbation of brain pathology after partial restraint in hypertensive rats following $\mathrm{SiO}_{2}$ nanoparticles exposure at high ambient temperature. Mol Neurobiol. 2013;48(2):368-379.

38. Wu J, Ding T, Sun J. Neurotoxic potential of iron oxide nanoparticles in the rat brain striatum hippocampus. Neurotoxicology. 2013;34:243-253.

39. Sun $H$, Chen $X$, Chen $D$, et al. Influences of surface coatings and components of FePt nanoparticles on the suppression of gliomas cell proliferation. Int J Nanomedicine. 2012;7:3295-3307.

40. Zhang K, Yu Y, Sun S. Facile synthesis L-cysteine capped CdS:Eu quantum dots and their $\mathrm{Hg}^{2+}$ sensitive properties. Appl Surface Sci. 2013;276:333-339. 
41. Aryal S, B K C R, Dharmaraj N, Bhattarai N, Kim CH, Kim HY. Spectroscopic identification of S-Au interaction in cysteine capped gold nanoparticles. Spectrochim Acta A Mol Biomol Spectrosc. 2006;63(1):160-163.

42. Chandra S, Saleem H, Sebastian S, Sundaraganesan N. The spectroscopic (FT-IR, FT-Raman), NCA, first order hyperpolarizability, NBO analysis, HOMO and LUMO analysis of L-cysteine by ab inito HF and density functional method. Spectrochim Acta A Mol Biomol Spectrosc. 2011;78(5):1515-1524.

43. Zhu X, Mason TG. Nanoparticle size distributions measured by optical adaptive-deconvolution passivated-gel electrophoresis. J Colloid Interface Sci. 2014;435:67-74.
44. Wu XW, Liu C, Li L, Jones P, Chantrell RW, Weller D. Nonmagnetic shell in surfactant-coated FePt nanoparticles. J Appl Phys. 2004; 95(11);6810-6812.

45. Aslam M, Fu L, Li S, Dravid VP. Silica encapsulation and magnetic properties of FePt nanoparticles. J Colloid Interface Sci. 2005;290;444-449.

46. Yu ACC, Mizuno M, Sasaki Y, Kondo H. Atomic composition effect on the ordering of solution-phase synthesized FePt nanoparticle films. Appl Phys Lett. 2004;85(25);6242-6244.
International Journal of Nanomedicine

\section{Publish your work in this journal}

The International Journal of Nanomedicine is an international, peerreviewed journal focusing on the application of nanotechnology in diagnostics, therapeutics, and drug delivery systems throughout the biomedical field. This journal is indexed on PubMed Central, MedLine, CAS, SciSearch $®$, Current Contents $® /$ Clinical Medicine,

\section{Dovepress}

Journal Citation Reports/Science Edition, EMBase, Scopus and the Elsevier Bibliographic databases. The manuscript management system is completely online and includes a very quick and fair peer-review system, which is all easy to use. Visit http://www.dovepress.com/ testimonials.php to read real quotes from published authors.

Submit your manuscript here: http://www.dovepress.com/international-journal-of-nanomedicine-journal 